\title{
Soft X-Ray Laser Spectroscopy on Trapped Highly Charged Ions at FLASH
}

\author{
S. W. Epp, ${ }^{1, *}$ J. R. Crespo López-Urrutia, ${ }^{1}$ G. Brenner, ${ }^{1}$ V. Mäckel, ${ }^{1}$ P. H. Mokler, ${ }^{1}$ R. Treusch,,${ }^{2}$ M. Kuhlmann, ${ }^{2}$ \\ M. V. Yurkov, ${ }^{2}$ J. Feldhaus, ${ }^{2}$ J. R. Schneider, ${ }^{2}$ M. Wellhöfer, ${ }^{3}$ M. Martins, ${ }^{3}$ W. Wurth, ${ }^{3}$ and J. Ullrich ${ }^{1}$ \\ ${ }^{1}$ Max-Planck-Institut für Kernphysik, D-69117 Heidelberg, Germany \\ ${ }^{2}$ DESY, D-22607 Hamburg, Germany \\ ${ }^{3}$ Institut für Experimentalphysik, Universität Hamburg, D-22761 Hamburg, Germany
}

(Received 28 December 2006; published 1 May 2007)

\begin{abstract}
In a proof-of-principle experiment, we demonstrate high-resolution resonant laser excitation in the soft x-ray region at $48.6 \mathrm{eV}$ of the $2{ }^{2} S_{1 / 2}$ to $2{ }^{2} P_{1 / 2}$ transition of Li-like $\mathrm{Fe}^{23+}$ ions trapped in an electron beam ion trap by using ultrabrilliant light from Free Electron Laser in Hamburg (FLASH). High precision spectroscopic studies of highly charged ions at this and upcoming x-ray lasers with an expected accuracy gain up to a factor of a thousand, become possible with our technique, thus potentially yielding fundamental insights, e.g., into basic aspects of QED.
\end{abstract}

DOI: 10.1103/PhysRevLett.98.183001

Precision tests of fundamental theories have laid the foundations to our current understanding of nature. Laser spectroscopy, in particular, has in this regard [1] yielded crucial results in chemistry, biology, physics, and other disciplines. An outstanding example is the investigation of the $1 S-2 S$ transition energy in atomic hydrogen on an accuracy level of $1.8 \times 10^{-14}$ [2], one of the most accurate measurements ever performed on a physical quantity. However, laser spectroscopy has been severely limited beyond the UV and vacuum ultraviolet (VUV) spectral range [3-5] due to the lack of appropriate light sources. An extension to higher photon energies could result out of the application of high harmonics generation (HHG) from pulsed lasers or with free electron laser (FEL) technology. The new Free-Electron Laser in Hamburg (FLASH [6]) is the first in a series of upcoming FEL devices, like LCLS (Stanford) or XFEL (Hamburg), extending the spectral range from few $\mathrm{eV}$ up to tens of $\mathrm{keV}$, as illustrated in Fig. 1. Thus, for example, direct resonant laser spectroscopy on highly charged ions (HCI $[7,8]$ ), where oneelectron bound-bound transitions up to about $130 \mathrm{keV}$ exist, will become feasible. HCI constitute a dominant fraction of the visible matter in stars, supernovae, nearstellar clouds, shocks, and jets from active galactic nuclei-all explored with increasing efforts and unprecedented accuracy in most recent satellite missions like $\mathrm{XMM}$ and Chandra. Precise knowledge of the line spectrum of $\mathrm{HCI}$ is indispensable for the understanding of those objects as well as of hot earthbound plasmas like in fusion research, and for exploring the equation of state (EOS) of warm dense matter.

Moreover, their comparably simple electronic structure makes HCIs an ideal testing ground for theory. The few remaining electrons orbiting closely the nucleus of a heavy HCI experience extreme electromagnetic fields and fielddependent effects are strongly boosted by powers of the nuclear charge $Z$, the quantity determining the field strength: The binding energy scales with $Z^{2}$ and the Lamb shift, for example, already with $Z^{4}$. The latter is
PACS numbers: 32.30.-r, 12.20.Fv, 31.30.Jv, 42.62.Fi

governed by quantum electrodynamics (QED), the most precise theory in physics, the foundation of any quantum field theory and, therefore, of the standard model, which can be explored using HCI in the strongest stationary electromagnetic fields available, reaching $10^{18} \mathrm{~V} / \mathrm{m}$ on average for a single $1 S$ electron bound to a uranium nucleus. The standard perturbative QED approximations using $Z \alpha$ expansions have to be replaced by all-order rigorous calculations in the strong field case.

Because of the steep $Z$ scaling, a wavelength measurement on an $\mathrm{H}$-like $\mathrm{HCI}$ can probe certain physical effects with a relevance comparable to a many orders of magnitude more precise experiment using atomic hydrogen. In other words, with increasing precision of transition energies accessible now for HCI, unprecedented deep insights

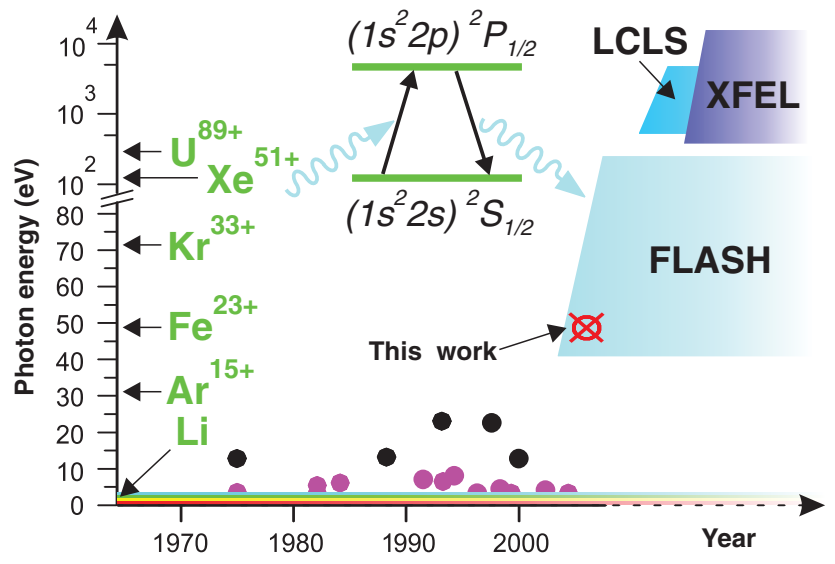

FIG. 1 (color). Photon energy range covered by FLASH and upcoming free electron laser facilities. The accessible spectral range for laser spectroscopy is extended by several orders of magnitude. For comparison, some benchmarking VUV laser spectroscopy experiments on transitions in neutral atoms [29] (black balls) are shown, as well as the most advanced laser spectroscopy measurements on transitions in multiply and highly charged ions (red balls) [30]. The transition energy $1 s^{2} 2 s-$ $1 s^{2} 2 p_{1 / 2}$ for some Li-like systems is given in (green). 
into the physics of bound systems at the strong field limit can be expected. Moreover, the advantageous scaling laws in $Z$ have been exploited for precision determination of the electron mass [9] and also inspired several schemes to study parity nonconservation (PNC) effects or drifts of the fine-structure constant $\alpha$ [10] involving HCI.

$\mathrm{HCI}$ up to even $\mathrm{H}$-like uranium have become available by accelerator techniques, in ion storage rings or in ion traps [11-16]. Overcoming the difficulties related to their production and preparation as a target at FELs will open a new chapter in x-ray precision spectroscopy, a field where accuracy had essentially stagnated in the recent decades due to limitations of wavelength determination by crystals, gratings, or solid state $x$-ray detectors. Presently, even the most precise results on $\mathrm{HCI}$ with $Z>20$ do not exceed a relative accuracy of $\Delta E / E \approx 1.5 \times 10^{-5}$ [17].

Here we report on the first demonstration of resonance fluorescence obtained by combining a soft x-ray free electron laser (FLASH) and a new electron beam ion trap (EBIT [13]) providing a HCI target. Critical difficulties resulting from very small excitation cross sections at those short wavelengths, photon fluxes still several orders of magnitude weaker than those typical for lasers in the visible, low radiation collection efficiency, and the achievable target density of HCI had to be overcome.

As a first objective, the transition between the $1 s^{2} 2 s$ ${ }^{2} S_{1 / 2}$ ground-level and $1 s^{2} 2 p^{2} P_{1 / 2}$ state (inset in Fig. 1), found in $\mathrm{Li}$ and every three-electron ion, was investigated in the Li-like iron $\mathrm{Fe}^{23+}$ ion, a very abundant ion, e.g., in solar flares. This transition is closely related to the Lamb shift in atomic $\mathrm{H}$ and one-electron ions. Since that was the key for the discovery of QED, it is not surprising that the investigated transition is also playing an important role in the formulation of few-electron QED in strong fields [1821]. In the isoelectronic sequence from $\mathrm{Li}$ to $\mathrm{U}^{89+}$, relative QED contributions to the total transition energy grow from $2 \times 10^{-5}$ to $1 \%$ at $Z=26$ or even $15 \%$ at $Z=92$. The transition energies of some Li-like systems are shown in Fig. 1 (in green). Most of them lay well within the FLASH spectral range.

The experiment was performed in a single photon resonant absorption scheme (Fig. 1), by tuning the laser over the ${ }^{2} S_{1 / 2}{ }^{2} P_{1 / 2}$ resonance around $48.6 \mathrm{eV}$ and registering the resonant fluorescence photon yield as a function of the laser wavelength. The lifetime of the excited level is $0.55 \mathrm{~ns}$ for the electronic dipole decay, and so excitation results in prompt emission of fluorescence radiation.

$\mathrm{Fe}^{23+}$ ions were produced in the EBIT and trapped as a cylindrical cloud. This cloud was brought to overlap with the FLASH beam (Fig. 2), which had with $300 \mu \mathrm{m}$ a comparable diameter. The laser light consisted of pulse trains of 30 single pulses of about $30 \mathrm{fs}$ length each (upper inset Fig. 2) with a $1 \mu$ s separation between them. The trains had a $5 \mathrm{~Hz}$ repetition rate, yielding a total of 150 laser pulses $/ \mathrm{s}$ with $\approx 2 \times 10^{12}$ photons each. So, the

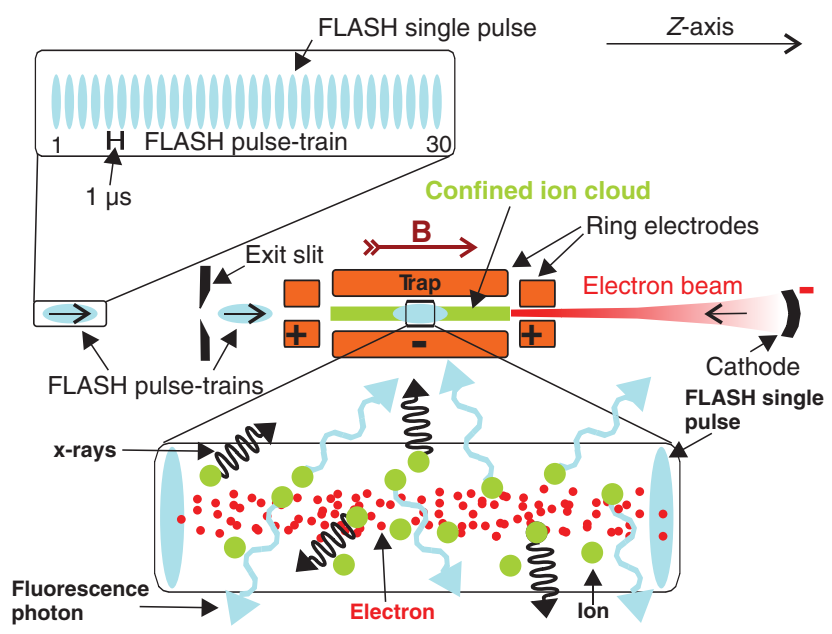

FIG. 2 (color). Ions produced by electron impact ionization of Fe atoms using a $450 \mathrm{~mA}, 5 \mathrm{keV}$ electron beam (compressed to a $50 \mu \mathrm{m}$ diameter by a $6 \mathrm{~T}$ coaxial magnetic field). Positive ions in the EBIT are confined radially by its negative space charge potential. Positively biased ring electrodes confine them longitudinally. The trapped HCIs form a cylindrical cloud of $50 \mathrm{~mm}$ length and 200-300 $\mu \mathrm{m}$ diameter with a density $\approx 10^{10}$ ions $/ \mathrm{cm}^{3}$. Ion charge states were monitored by x-ray detectors. Soft $x$ rays (light blue) from FLASH excited the trapped $\mathrm{Li}$-like $\mathrm{Fe}^{23+}$ ions. Fluorescence radiation was focused by x-ray mirrors on a microchannel plate detector (not depicted) with an overall photon detection efficiency of $\approx 10^{-4}$.

primary flux amounts to roughly $3 \times 10^{14}$ photons $/ \mathrm{s}$. Because of the self-amplified spontaneous emission (SASE) origin of the pulses, an average pulse train has a bandwidth of roughly $E / \Delta E \approx 50$ to 200 . This bandwidth was reduced by means of a scanning plane grating monochromator [22] with a maximum resolution up to 70000 , located at the PG2 beam line. Of course, depending on the desired resolution, the total photon flux is also diminished. As a compromise, a resolution $E / \Delta E \approx 2000$ at $48.6 \mathrm{eV}$ photon energy was chosen $\left(3 \times 10^{12}\right.$ photons/s, transmission efficiency included). The main advantage of this scheme is that the total photon flux of the FLASH beam is relativistically forward boosted into a narrow angular cone such that it can be made highly monochromatic with much smaller loss of efficiency than what is possible if the radiation emitted by the ions, or by any sample, into a $4 \pi$ solid angle is spectrally analyzed with a conventional spectrometer.

Photons were recorded in time coincidence with the FEL pulses as a function of the monochromator energy settings. Each pulse started a time window of $1 \mu \mathrm{s}$. Photons within this time window and their arrival times were registered. The monochromator was repeatedly scanned between 48.53 and $48.71 \mathrm{eV}$ in $5 \mathrm{meV}$ wide steps, with $3 \mathrm{~s}$ integration time on each one for every scan. Figure 3 shows the photon yield as a function of photon energy and arrival time, and its projection onto the photon energy axis. Establishing the overlap of ion cloud and laser beam turned 


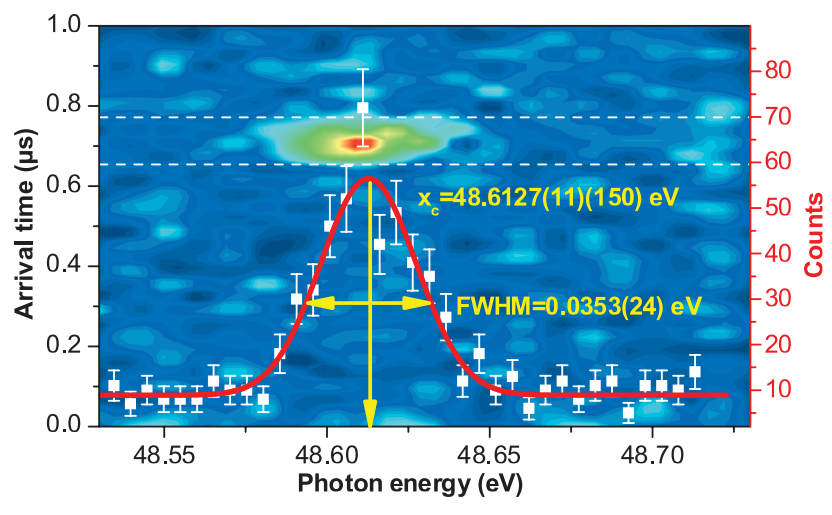

FIG. 3 (color). 2D plot (smoothed data, $5 \mathrm{meV} \times 0.016 \mu \mathrm{m}$ binning) of the fluorescence signal as a function of the photon arrival time relative to the FEL pulse (left $y$ axis, absolute scale arbitrary, excitation time does not match with ordinate zero) versus photon energy ( $x$ axis). Inset: Projection of the elevant band (white lines; white squares) onto the photon energy axis yielding the number of photons (right $y$ axis) as a function of the photon energy (see text). Red line: Gaussian fit.

out to be very time consuming: First, due to the small fluorescence signal and second due to demanding alignment of the $1500 \mathrm{~kg}$ heavy EBIT to the FLASH beam in steps of a few microns. Although these problems restricted the final run duration to $1987 \mathrm{~s}$ only, a resonance signal in time coincidence with excitation containing about 370 true counts over a $5 \sigma$ interval became clearly visible. The time width of this signal, due to the limited time resolution of the detector system, is $90 \mathrm{~ns}$ FWHM. The delay represented on Fig. 3 is purely instrumental and can be varied arbitrarily between 0 and $1 \mu \mathrm{s}$. The coincidence of excitation and fluorescence radiation is of essential importance to suppress background. A Gaussian fit of the data (Fig. 3) yields a transition energy of $48.6127(11)_{\text {stat }} \times$ $(150)_{\text {syst }} \mathrm{eV}$. So far, the absolute value suffers under the preliminary calibration uncertainty $( \pm 0.0150 \mathrm{eV})$ of the PG2 beam line monochromator. It will be reduced considerably by an adequate calibration of that instrument in the near future.

Several tests excluded possible spurious contributions. The signal disappeared when (i) there was no spatial overlap between ions and photons, (ii) the $\mathrm{Fe}$ injection was switched off, so background from scattered photons was also ruled out, and (iii) the trap potential was set to expel the positive ions. The identification of the lines recorded by an additional independent instrument, a flat-field grating spectrometer, ensured that in the energy range scanned the line of interest was present and not blended. Analysis of this instrument's spectra yielded an independent value for the level energy difference of 48.5982(8) eV, in fair agreement with the most accurate published result [23] of 48.5997(9) eV.

In Fig. 4 we compare the relative (statistical) accuracy achieved in this first soft $\mathrm{x}$-ray laser resonance fluorescence

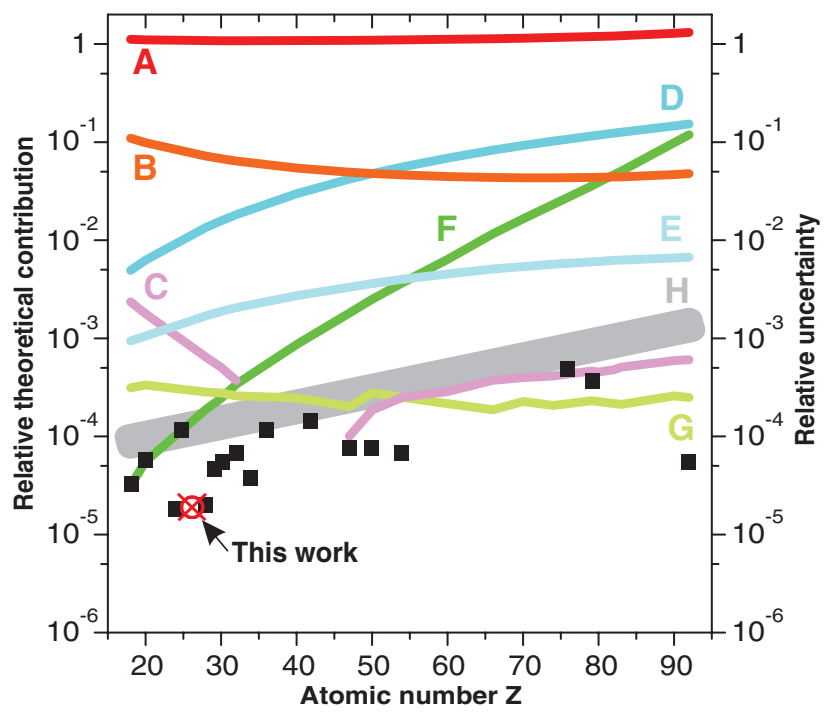

FIG. 4 (color). Different relative contributions (left scale) to the total $1 s^{2} 2 s-1 s^{2} 2 p_{1 / 2}$ transition energy in Li-like HCI as a function of $Z$ [18-20]. Interelectronic: (A) one, (B) two, and (C) three virtual photon exchange between the valence and core electrons. Radiative corrections: (D) one-loop self energy + vacuum polarization (H-like), (E) screening of (D) by core electrons. Nuclear corrections: $(F)$ finite nuclear size, $(\mathrm{G})$ relativistic recoil. Total relative uncertainties (right scale): $(\mathrm{H})$ theoretical; (black squares) experimental ([15], [17,18], [23$26]$ and references therein), and (red cross) this work.

experiment with that of a selection of the most precise published results for different Li-like HCI (cf. right scale). Various nonlaser spectroscopic techniques have been applied: beam-foil methods [24,25], emission from tokamaks [17], storage rings [26], and EBITs [15], providing the most accurate tests of bound state QED effects in strong fields up to now. With $22 \mathrm{ppm}$ (statistical) accuracy, accomplished in spite of the short data acquisition time, our soft X-ray laser method challenges already the most accurate conventional measurements.

The relative accuracy achieved here is already higher than the theoretical uncertainties. The various theoretical contributions to the considered transition energy are depicted in Fig. 4 (cf. left ordinate). The contributions A, B, and $\mathrm{C}$ concern the interelectron interaction (nonradiative QED with one, two, and three photon exchange, respectively). The true radiative contributions are $\mathrm{D}$ and $\mathrm{E}$, where $\mathrm{D}$ comprises the single electron one-loop terms self energy (SE) and vacuum polarization (VP) - and E the corresponding screening terms, respectively. We note that in both cases VP is in the order of $10 \%$ of the SE part. True two-loop QED terms-SE-SE, SE-VP, VP-VP, $\mathrm{S}(\mathrm{VP}) \mathrm{E}, \ldots$ - . approach the order of the screened VP contributions (10\% of E) and are on the order of the present theoretical uncertainties indicated by the gray band $\mathrm{H}$. The influence of the finite extension of the nucleus and its recoil are indicated by $\mathrm{F}$ and $\mathrm{G}$. 
Present experimental accuracy allows us already to verify the leading two-photon QED terms. Any further increase in experimental precision (Fig. 4) provides a systematic sensitivity improvement: namely, one additional photon in the QED graphs for any accuracy gain of $1 / \alpha(\approx 137)$. Hence, so far uncalculated two-loop [20,21,27] and higher order QED contributions [28], as well as model dependent nuclear-size and polarization effects, which are limiting the currently pursued tests of "pure" QED will be revealed. The future use of shortlived radioactive HCI will boost nuclear effects and allows for studies along extended isotopic sequences. This could lead to tests of QED beyond the external-field approximation [20], which is the foundation of present $a b$ initio QED calculations of the electronic structure in HCI.

In conclusion, with our laser spectroscopic approach we have reached a statistical accuracy of $22 \mathrm{ppm}$ for the transition energy in only half an hour acquisition time, at a moderate laser bandwidth of $500 \mathrm{ppm}$. The immediate potential becomes obvious, by considering that existing monochromators could reduce the FEL bandwidth by a factor of 100 down to $5 \mathrm{ppm}$. Although a wavelength calibration in the soft x-ray range on this accuracy level is by no means trivial, we plan an in situ calibration with the EBIT by utilizing well-known transitions in light $\mathrm{H}$-like ions. A short term potential improvement of 2 orders of magnitude in accuracy can be expected. At some point in the future, the employment of a soft x-ray frequency comb as suggested by Udem [3] could deliver ultimate precision in this spectral range as well.

In the near future, photon energies up to $10 \mathrm{keV}$ will become available with unprecedented flux at upcoming x-ray FELs like the Stanford Linear Coherent Light Source (LCLS) or the European XFEL (Fig. 1), allowing to access all transitions in Li-like ions as well as $n=1$ to $n=2$ transitions in He- and H-like systems up to $Z \approx 70$ (5th harmonic). Because of the 20-fs timing of these devices, with envisioned attosecond resolution, lifetime measurements in a hitherto inaccessible time-energy regime will become feasible. The present experiment demonstrates laser spectroscopy in this spectral range on bound state transitions in HCI by combining the FEL and EBIT technologies, showing the wide range of possibilities opened up to research by soft x-ray lasers.

We acknowledge help from: V. Kocharyan, E.A. Schneidmiller, and the whole FLASH-team; C. Kaiser, K. Bechberger, N. Müller, and T. Busch (MPI-K) for technical support, our interns D. Menon, L. Schmöger, and B.L. Schmitt. Funding was provided through the MIDFEL-Initiative.
*Electronic address: epp@mpi-hd.mpg.de

[1] T. W. Hänsch and H. Walther, Rev. Mod. Phys. 71, S242 (1999).

[2] M. Niering et al., Phys. Rev. Lett. 84, 5496 (2000).

[3] T. Udem, Science 307, 364 (2005).

[4] K. S. E. Eikema et al., Phys. Rev. Lett. 76, 1216 (1996).

[5] S. D. Bergeson et al., Phys. Rev. Lett. 80, 3475 (1998).

[6] V. Ayvazyan et al., Eur. Phys. J. D 37, 297 (2006).

[7] J. D. Gillaspy, J. Phys. B 34, R93 (2001).

[8] I. Martinson, Rep. Prog. Phys. 52, 157 (1989).

[9] P. J. Mohr and B. N. Taylor, Rev. Mod. Phys. 77, 1 (2005).

[10] O. Y. Andreev, L. N. Labzowsky, G. Plunien, and G. Soff, Phys. Rev. Lett. 94, 243002 (2005).

[11] J. Schweppe et al., Phys. Rev. Lett. 66, 1434 (1991).

[12] A. Gumberidze et al., Phys. Rev. Lett. 94, 223001 (2005).

[13] R.E. Marrs, P. Beiersdorfer, and D. Schneider, Phys. Today 47, No. 10, 27 (1994).

[14] R.E. Marrs, S. R. Elliott, and D. A. Knapp, Phys. Rev. Lett. 72, 4082 (1994).

[15] P. Beiersdorfer, H. Chen, D. B. Thorn, and E. Träbert, Phys. Rev. Lett. 95, 233003 (2005).

[16] K. Hosaka et al., Phys. Rev. A 69, 011802 (2004).

[17] J. Sugar, V. Kaufman, and W. L. Rowan, J. Opt. Soc. Am. B 10, 13 (1993).

[18] V. A. Yerokhin et al., Phys. Rev. A 64, 032109 (2001).

[19] O. Y. Andreev, L. N. Labzowsky, and G. Plunien, Phys. Rev. A 67, 012503 (2003).

[20] V. M. Shabaev et al., Int. J. Mass Spectrom. 251, 109 (2006).

[21] V. A. Yerokhin, P. Indelicato, and V. M. Shabaev, Phys. Rev. Lett. 97, 253004 (2006).

[22] M. Martins et al., Rev. Sci. Instrum. 77, 115108 (2006).

[23] J. Reader, J. Sugar, N. Acquista, and R. Bahr, J. Opt. Soc. Am. B 11, 1930 (1994).

[24] D. Feili et al., Phys. Rev. A 62, 022501 (2000).

[25] E. Hinnov and B. Denne, Phys. Rev. A 40, 4357 (1989).

[26] C. Brandau et al., Phys. Rev. Lett. 91, 073202 (2003).

[27] J. Sapirstein and K. T. Cheng, Phys. Rev. A 64, 022502 (2001).

[28] O. M. Zherebtsov, V.M. Shabaev, and V.A. Yerokhin, Phys. Lett. A 277, 227 (2000).

[29] T. W. Hänsch et al. Phys. Rev. Lett. 34, 307 (1975); S. Chu et al., Phys. Rev. Lett. 80, 3475 (1998); K. S. E. Eikema et al. [4]; S. D. Bergeson et al. [5]; M. Niering et al. [2].

[30] H. W. Kugel et al., Phys. Rev. Lett. 35, 647 (1975); O. R. Wood et al., Phys. Rev. Lett. 48, 398 (1982); P. Pellegrin et al., Phys. Rev. Lett. 49, 1762 (1982); H. D. Sträter et al., Phys. Rev. A 29, 1596 (1984); T. P. Dinneen et al., Phys. Rev. Lett. 66, 2859 (1991); T. J. Scholl et al., Phys. Rev. Lett. 71, 2188 (1993); I. Klaft et al., Phys. Rev. Lett. 73, 2425 (1994); E. G. Myers et al., Phys. Rev. Lett. 76, 4899 (1996); P. Seelig et al., Phys. Rev. Lett. 81, 4824 (1998); E. G. Myers et al., Phys. Rev. Lett. 82, 4200 (1999); M. Redshaw and E. G. Myers, Phys. Rev. Lett. 88, 023002 (2001); K. Hosaka et al. Phys. Rev. A 69, 011802 (2004). 